\title{
BANDA GÁSTRICA AJUSTÁVEL LAPAROSCÓPICA: ALTERNATIVA TÉCNICA
}

\author{
LAPAROSCOPIC ADJUSTABLE GASTRIC BANDING: \\ TECHNICAL ALTERNATIVE
}

\author{
Pablo Roberto Miguel, TCBC-RS ${ }^{1}$ \\ Marcus Reusch ${ }^{1}$ \\ André Luiz Moreira da Rosa ${ }^{1}$
}

\section{INTRODUÇÃO}

A prevalência de obesidade tem aumentado marcadamente durante as últimas duas décadas ${ }^{1}$. A obesidade mórbida, definida como índice de massa corporal (IMC) maior que $40 \mathrm{~kg} / \mathrm{m}^{2}$, está associada com um número significativo de condições de co-morbidade e redução de expectativa de vida ${ }^{2}$.

Devido ao reconhecimento de os tratamentos conservadores para obesidade mórbida estarem associados com quase $100 \%$ de insucesso a longo prazo, a demanda de tratamento cirúrgico tem se expandido rapidamente com a ajuda das técnicas laparoscópicas ${ }^{3}$.

Conscientes das dificuldades técnicas óbvias, que são estabelecidas no período transoperatório pelo excesso de gordura intra-abdominal, procuramos criar uma técnica mais fácil e segura. A forma fragmentada em duas etapas de passagem da banda pelo estômago é facilmente padronizável e reprodutível.

\section{TÉCNICA}

Após estabelecer um pneumoperitônio com aproximadamente $16 \mathrm{mmHg}$ e campo operatório adequado, iniciamos o procedimento abrindo o peritônio do pilar diafragmático esquerdo. Em seguida abrimos o pequeno omento, à direita do estômago, e fazemos o isolamento do estômago superior dissecando o tecido retrogástrico junto à união dos pilares diafragmáticos, começando pelo pilar direito.
Criamos neste ponto o túnel retrogástrico por onde será tracionado o cateter da banda. Neste momento, o estômago está isolado pelo cateter, mas ainda mantém toda gordura do pequeno omento em conjunto. Inicia-se então o segundo passo da colocação da banda. Um balão com $30 \mathrm{ml}$ de volume é inflado dentro do estômago e tracionado até a junção gastroesofágica para moldar o novo estômago. Abaixo do balão, na margem direita do estômago, isola-se toda gordura do pequeno omento e traciona-se o cateter da banda de forma a ficar com o estômago isolado sem excesso de gordura na sua circunferência (Figura 1). Para finalizar, dois pontos, com fio inabsorvível 2-0 unindo estômago com estômago por sobre a banda fechada, e um ponto, unindo o fundo gástrico ao pilar esquerdo, são aplicados.

A técnica descrita foi utilizada em todos os pacientes da casuística (60 pacientes do estudo). O tempo operatório médio foi de 52,0 minutos. O período de acompanhamento pós-operatório médio foi de cinco meses. Dos primeiros 18 casos foi possível medir a queda do IMC médio em 120 dias, que diminuiu de 41,5 para 32,6 . A permanência hospitalar média foi de 24 horas, entretanto a rotina atual é de 12 horas. Chevallier et al. ${ }^{4}$ referem uma permanência média de 5,4 dias.

Ocorreram complicações em sete casos $(11,6 \%)$. Houve um caso de deslizamento do fundo gástrico através da banda (slippage), exigindo reintervenção por laparoscopia. Seis pacientes apresentaram problemas com os orifícios dos trocartes (seromas ou infecções). Não ocorreram complicações respiratórias.

\section{Cirurgiões do Hospital Mãe de Deus}

Recebido em 7/6/2000

Aceito para publicação em 5/4/2001

Trabalho realizado no Serviço de Cirurgia Geral do Hospital Mãe de Deus - Porto Alegre - RS. 


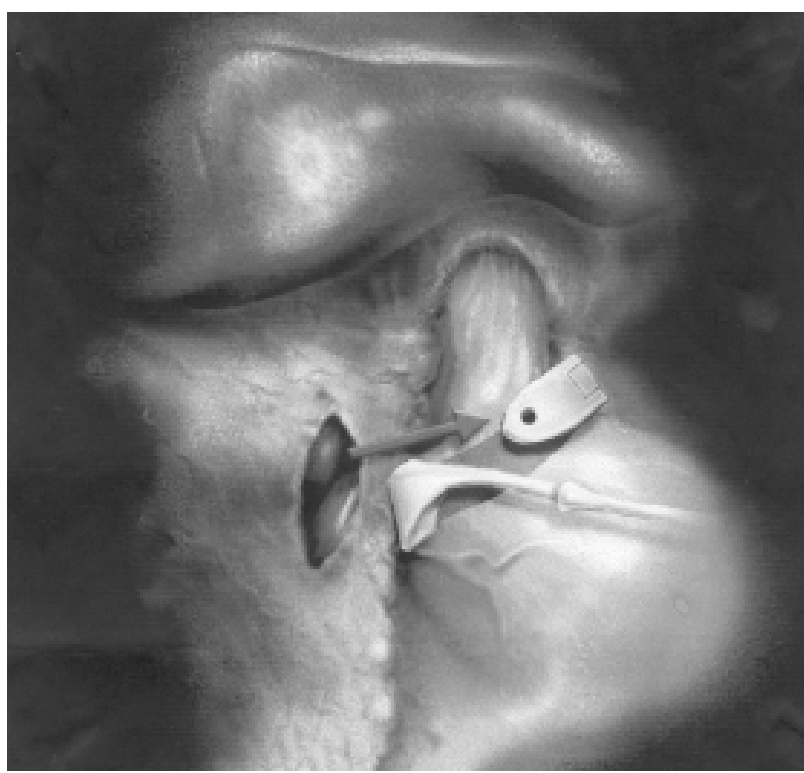

Figura 1 - Esquema da Técnica para colocação da banda gástrica.

\section{DISCUSSÃo}

A técnica de colocação da banda aqui apresentada permitiu obter tempo cirúrgico baixo, comparado aos 80 minutos encontrados no trabalho de De La Garza et al ${ }^{5}$. A segunda passagem do cateter da banda entre a parede do estômago e a gordura, à direita, deixa-a com uma fixação natural, auxiliando na prevenção de deslizamento (slippage). O primeiro passo é feito junto aos pilares diafragmáticos, em uma região anatômica íntima do cirurgião acostumado aos procedimentos de fundoplicatura posterior. O segundo passo, que consiste em passar a banda junto à parede do estômago, fica facilitado, uma vez que ela já passou pelo espaço retrogástrico no primeiro momento. É uma técnica que utiliza espaços anatômicos avasculares e evita a passagem cega de instrumentos, prevenindo lesões viscerais durante o procedimento. Acreditamos ser uma alternativa técnica segura para o tratamento da obesidade mórbida com banda gástrica laparoscópica.

\begin{abstract}
In these paper we are presenting a technical alternative to laparoscopic adjustable gastric banding. From January 1999 to April 2000, 60 patients with mean body mass index (BMI) of 40,7 kg/m2 underwent laparoscopic adjustable gastric banding. The new technique is performed in two steps. In the first step, an isolation instrument (laparoscopic finger) is inserted through the lesser sac, next to the junction of diaphragmatic crura, including the lesser omentum in order to pull the band catheter. The second step separates the lesser omentum from the right side of the stomach.There was no mortality and the morbidity was 11,6\% (1 slippage of the band and 6 trocar port seroma). The new technique was performed in all patients with no conversion to open procedure. We didn't have respiratory complications. This technical alternative is safe and easily performed, helping to prevent transoperative perforations.
\end{abstract}

Key Words : Obesity surgery; Laparoscopic surgery; Gastric banding; Bariatric surgery.

\section{REFERÊNCIAS}

1. Forsell P, Hellers G.: The Swedish adjustable gastric banding for morbid obesity: 9 years experience and a 4 year follow-up of patients operated with a new adjustable band. Obes Surg 1997;7:345-51.

2. Favretti F, Cadière GB, Segato G, et al: Laparoscopic adjustable silicone gastric banding: how to avoid complications. Obes Surg 1997; 7:352-8.

3. Chelala E, Cadière GB, Favretti F,et al: Conversions and complications in 185 laparoscopic adjustable silicone gastric banding cases. Surg Endosc 1997; 11:26871.

4. Chevallier JM, Zinzindohoue F, Blanche J, et al: 150 LapBand since 2 years: selection, learning curve, and first results. Obes Surg 1999; 9:329-30
5. De La Garza J, Vazquez J , Saucedo P.: Laparoscopic gastric banding. Swedish adjustable gastric band. First 100 cases in Mexico. Obes Surg 1999; 9: 328.

Endereço para correspondência:

Dr. Pablo Roberto Miguel

Rua Costa,.30 conj. 502

90110-270 - Porto Alegre - RS

Tel./Fax.: (51) 231-4407 